\title{
Survival is associated with complete response on MRI after neoadjuvant chemotherapy in ER-positive HER2-negative breast cancer
}

Claudette E. Loo ${ }^{1 *}$, Lisanne S. Rigter ${ }^{2}$, Kenneth E. Pengel', Jelle Wesseling ${ }^{3}$, Sjoerd Rodenhuis², Marie-Jeanne T. F. D. Vrancken Peeters ${ }^{4}$, Karolina Sikorska ${ }^{5}$ and Kenneth G. A. Gilhuijs ${ }^{1,6}$

\begin{abstract}
Background: Pathological complete remission ( $p C R$ ) of estrogen receptor (ER)-positive/human epidermal growth factor receptor 2 (HER2)-negative breast cancer is rarely achieved after neoadjuvant chemotherapy (NAC). In addition, the prognostic value of pCR for this breast cancer subtype is limited. We explored whether response evaluation by magnetic resonance imaging (MRI) is associated with recurrence-free survival after NAC in ERpositive/HER2-negative breast cancer.

Methods: MRI examinations were performed in 272 women with ER-positive/HER2-negative breast cancer before, during and after NAC. MRI interpretation included lesion morphology at baseline, changes in morphology and size, and contrast uptake kinetics. These MRI features, clinical characteristics and final pathology were correlated with recurrence-free survival.

Results: The median follow up time was 41 months. There were 35 women with events, including 19 breast-cancerrelated deaths. On multivariable analysis, age younger than 50 years (hazard ratio $(H R)=2.55,95 \%$ confidence interval (Cl) $1.3,5.02, p=0.007)$, radiological complete response after NAC (HR $=14.11, \mathrm{Cl} 1.81,1818 ; p=0.006)$ and smaller diameters of washout/plateau enhancement at MRI after NAC ( $H R=1.02, \mathrm{Cl} 1.00,1.04, p=0.036)$ were independently associated with best recurrence-free survival. Pathological response was not significant; $\mathrm{HR}=2.12, \mathrm{Cl} 0.86,4.64, p=0.096$.

Conclusions: MRI after NAC in ER-positive/HER2-negative tumors may be predictive of recurrence-free survival. A radiological complete response at MRI after NAC is associated with an excellent prognosis.
\end{abstract}

Keywords: Breast cancer, Neoadjuvant chemotherapy, Magnetic resonance imaging, Recurrence-free survival, Estrogen receptor

\section{Background}

Neoadjuvant chemotherapy (NAC) for breast cancer has been shown to be equally effective as postoperative chemotherapy in terms of disease-free and overall survival [1-4]. Several markers are routinely employed to predict treatment outcome and to select therapy [5-7]. The most frequently used include the estrogen receptor

\footnotetext{
* Correspondence: c.loo@nki.nl

'Division of Diagnostic Oncology (Department of Radiology), The Netherlands Cancer Institute - Antoni van Leeuwenhoek Hospital, Plesmanlaan 121, Amsterdam 1066 CX, The Netherlands

Full list of author information is available at the end of the article
}

(ER), the progesterone receptor (PR) and the human epidermal growth factor receptor 2 (HER2). Three major breast cancer subtypes are easily distinguished by immunohistochemical assessment (IHC): triple-negative (ER, PR and HER2-negative), HER2-positive (HER2-positive (ER and PR may be positive or negative)) and ERpositive/HER2-negative (ER-positive, HER2-negative (PR may be positive or negative)) [8, 9]. These immunohistochemical subtypes correspond roughly to the molecular subtypes, basal-like, HER2-enriched and luminal, respectively [10]. Subtyping of typically heterogeneous breast cancer in these three groups may improve 
understanding of tumor response and outcome and may result in optimized strategies for patient-tailored treatment $[11,12]$.

Even within these subgroups, the response to and outcome after chemotherapy vary widely. Pathologically confirmed complete remission ( $\mathrm{pCR}$ ) or minimal disease $[13,14]$ after chemotherapy is associated with diseasefree and overall survival $[1,2,15,16]$. More recently, however, it has been shown that this relationship is absent for luminal A tumors [17], which comprise approximately half of the tumors that express the ER but which do not contain a HER2 gene amplification. Nevertheless, pCR is often used as a surrogate marker to predict longterm outcome in this subgroup. Of patients with ERpositive/HER2-negative tumors only a small fraction will achieve pCR, while the prognosis is better than that of triple-negative breast cancer [17]. Therefore pCR after NAC in ER-positive/HER2-negative tumors is certainly not a practical prognostic indicator. It is possible that dynamic contrast-enhanced magnetic resonance imaging (MRI), which visualizes functional properties of the tumor such as those associated with angiogenesis, may be used as a practical prognostic indicator.

The benefit of MRI over other imaging modalities for monitoring response during and after NAC has been extensively reported [18-21]. Also prediction of pathological response after NAC by MRI has been extensively studied [22-24]. A recent published study evaluated volumetric MRI for predicting recurrence-free survival after NAC in patients with breast cancer [25]. However, the role of MRI after NAC in predicting survival in patients with ER-positive/HER-2negative tumors in particular has not yet been completely assessed. The purpose of this study was to explore whether MRI is associated with recurrence-free survival after neoadjuvant chemotherapy in ER-positive/HER2-negative breast cancer.

\section{Methods}

\section{Selection of patients}

Patients between 18 and 70 years of age with pathologically proven invasive ER-positive/HER2-negative breast cancer $>3 \mathrm{~cm}$ in size and/or at least one tumor-positive lymph node were offered NAC. All patients received NAC between January 2000 and June 2012, and all either took part in a single-institution clinical trial (approved by the Medical Research Ethics Committee of the Netherlands Cancer Institute), or were treated off study according to the standard arm of the trial $[26,27]$. The institutional review board had approved the study protocols and informed consent was obtained from all patients. Only patients with ER-positive/HER2-negative tumors based on immunohistochemical assessment without a prior history of breast cancer were included in this analysis. Only patients who had undergone MRI before (baseline), during (after three courses) and after NAC and who underwent surgery after NAC were included.

\section{Treatment}

Four different regimens of NAC were employed [26, 27]. Between 2000 and 2004 patients were randomized to receive either six cycles of treatment AC or six cycles of treatment $\mathrm{AD}$, with $\mathrm{AC}$ being considered as standard treatment. AC consisted of doxorubicin $60 \mathrm{mg} / \mathrm{m}^{2}$ and cyclophosphamide $600 \mathrm{mg} / \mathrm{m}^{2}$ every three weeks, whereas patients in the $\mathrm{AD}$ arm were treated with six cycles of doxorubicin $50 \mathrm{mg} / \mathrm{m}^{2}$ and docetaxel $75 \mathrm{mg} /$ $\mathrm{m}^{2}$. After 2004, patients started with three courses of ddAC (doxorubicin $60 \mathrm{mg} / \mathrm{m}^{-2}$ and cyclophosphamide $600 \mathrm{mg} / \mathrm{m}^{-2}$ on day 1 , every 14 days, with PEGfilgrastim on day 2). When an unfavorable response was noted on MRI (defined as a reduction $<25 \%$ in the largest diameter of the tumor plateau/washout enhancement [28]) after three courses of treatment, chemotherapy was switched to a (theoretically) non-cross-resistant regimen. In such a case, three courses of ddAC were followed by three courses of docetaxel and capecitabine (DC, docetaxel $75 \mathrm{mg} / \mathrm{m}^{-2}$ on day 1 , every 21 days and capecitabine $2 \times 1000 \mathrm{mg} / \mathrm{m}^{-2}$ on days $\left.1-14\right)$. In the case of a favourable response on MRI, chemotherapy was continued with three further courses of ddAC.

After the last course of chemotherapy, all patients underwent surgery (breast-conserving surgery or mastectomy with or without axillary lymph node dissection), post-operative external beam radiation therapy, and adjuvant endocrine therapy, according to standard guidelines.

\section{MRI and evaluation}

Initially MRI was performed on a $1.5 \mathrm{~T}$ Magnetom Vision scanner with a dedicated bilateral phased array breast coil (Siemens, Erlangen, Germany). From April 2007 MRI was performed on a 3.0 T Achieva scanner with a dedicated 7-element sense breast coil (Philips Medical Systems, Best, The Netherlands). Images were acquired with the patient in the prone position and with both breasts imaged simultaneously. The standard dynamic protocol started with an unenhanced coronal 3D fast field echo (FFE) (thrive) sense T1-weighted sequence. A bolus $(14 \mathrm{~mL})$ of contrast containing gadolinium $(0.1 \mathrm{mmol} / \mathrm{kg})$ was administered intravenously at 3 $\mathrm{mL} / \mathrm{s}$ using a power injector followed by a bolus of 30 $\mathrm{mL}$ of saline solution. Subsequently, dynamic imaging was performed in five consecutive series at 90-s intervals. The voxel size was $1.21 \times 1.21 \times 1.69 \mathrm{~mm}^{3}(1.5 \mathrm{~T})$ or $1.1 \times 1.1 \times 1.2 \mathrm{~mm}^{3}(3.0 \mathrm{~T})$. The following scanning parameters were used: acquisition time $90 \mathrm{~s}(1.5 \mathrm{~T}$ and 
3.0 T); repetition time (TR)/echo time (TE): 8.1/4.0 (1.5 $\mathrm{T})$ or $4.4 / 2.3(3.0 \mathrm{~T})$; flip angle $20^{\circ}(1.5 \mathrm{~T})$ or $10^{\circ}(3.0 \mathrm{~T})$; field of view (FOV) $310(1.5 \mathrm{~T})$ or $360(3.0 \mathrm{~T})$.

Breast MR images were interpreted using a viewing station that permitted simultaneous viewing of two series reformatted and linked in three orthogonal directions [29]. The viewing station displays all imaging series (unenhanced and contrast-enhanced), subtraction images at 90-s intervals and maximum intensity projection (MIP) of both breasts. The displayed images were also color coded, representing different levels and curve types of enhancement. Specifically, the color indicated the shape of the time-signal intensity (contrast enhancement) curve at each pixel location [30]: type I (i.e., persistent enhancement $>10 \%$ after the first post-contrast image), type II (i.e., plateau enhancement between $-10 \%$ and $+10 \%$ during late enhancement), and type III (i.e., washout kinetics resulting in $>10 \%$ signal decrease during late enhancement) [30]. These colors were coded yellow, light red and dark red, respectively, where initial enhancement (90 s) equaled or exceeded $100 \%$ and green, light blue and dark blue, respectively, where initial enhancement was between $50 \%$ and $100 \%$. The viewing station was developed in close collaboration with the breast radiologists at the Netherlands Cancer Institute. The radiologists have been using the system since 2000 .

The MR images were assessed by four breast radiologists, who were unaware of the outcome. The patients were randomly distributed among the radiologists for assessment. The MR images before (baseline), during and after chemotherapy were analyzed by the same radiologist in one session to ensure interpretive consistency. Temporal and morphologic characteristics of contrast uptake were scored as previously described [28]. In short, tumor extent, morphology and relative enhancement were assessed during initial enhancement (90 s) and late enhancement (450 s) on all subsequent MRI scans.

The extent of the tumor was assessed by its largest diameter in three reformatted planes (sagittal, axial and coronal) at initial and at late (washout/plateau) enhancement separately. If a non-mass (diffuse) enhancement or multifocal disease was visible, the total area including non-enhancing breast tissue between lesions was measured on MIP images. The largest value of the three diameters was recorded. The percentage difference in largest tumor diameter between subsequent MRI scans was also assessed, both at initial and at late enhancement. Supported by the color coding, the area within the tumor with the strongest contrast uptake at initial and at late enhancement was determined. Measurement of the signal intensity (initial and late enhancement) was performed manually by placing a region of interest in the most malignant area (dark red) and moving the cursor in this area to find the most malignant values, in real time (in percentages). Morphology of the enhancing tumor was scored in three groups: unifocal mass, multifocal mass and non-mass (diffuse) enhancement [31]. On MRI during and after NAC the pattern of tumor reduction was denoted in five categories: shrinking mass, diffuse decrease, reduction to small foci, no enhancement and no change.

Complete absence of contrast enhancement in the original tumor bed on MRI after NAC was defined as radiological complete response. Consequently, evidence of small enhancing foci in the original tumor bed was considered as residual enhancing tumor.

\section{Histopathologic analysis}

Prior to NAC at least three 14-G ultrasound-guided core biopsies of the breast tumor were taken. Subsequently, most tumors were marked with a radiopaque marker. ER and PR status were determined by immunohistochemical assessment and considered positive if $\geq 10 \%$ of nuclei stained positive, and HER2 status was assessed by scoring the intensity of membrane staining. Tumors with a score of $3+$ (strong homogeneous staining) were considered positive. In the case of $2+$ scores (moderate homogeneous staining) chromogenic in situ hybridization (CISH) was used to determine HER2 amplification (gene copy number of six or more per tumor cell). For this study ERpositive/HER2-negative tumors were selected.

\section{Pathologic response}

Three common definitions of pCR were used: (1) no residual invasive tumor in the breast (ypT0/is) [15, 32], (2) no residual invasive tumor in the breast or axilla (ypT0/ isN0) [33] and (3) a near-complete response, indicating the presence of only a small number of scattered tumor cells in the breast (ypT < mic) [14].

\section{Statistical analysis}

The primary endpoint was recurrence-free survival (RFS), defined according to the standardization of events and endpoints (STEEP) criteria [34]. According to this definition an event is either a local, regional or distant breast cancer recurrence or death due to any cause. Second primaries (including contralateral breast cancer) were not considered an event. The final data were collected in September 2014, and patients for whom no event had occurred were censored at the last date of being seen alive.

The median length of follow up was calculated using the reverse Kaplan-Meier approach. Patient characteristics are presented in tables as medians (percentiles) for continuous variables and frequencies for categorical variables. All clinical variables were analyzed as categorical predictors (Table 1). The MRI characteristics were 
Table 1 Univariable Cox proportional hazard analysis of relationship between clinical variables and recurrence-free survival

\begin{tabular}{|c|c|c|c|c|c|}
\hline \multirow[b]{2}{*}{ Variable } & \multirow[b]{2}{*}{ Number of patients } & \multirow[b]{2}{*}{ Number of events } & \multicolumn{3}{|c|}{ Recurrence-free survival } \\
\hline & & & $P$ value & Hazard ratio & $95 \% \mathrm{Cl}$ \\
\hline Tumor (T) stage prior to NAC & & & 0.731 & & \\
\hline $\mathrm{T} 1$ & 28 & 1 & & & \\
\hline $\mathrm{T} 2$ & 149 & 19 & & 2.42 & $0.32,18.16$ \\
\hline T3 & 79 & 12 & & 2.7 & $0.35,20.99$ \\
\hline $\mathrm{T} 4$ & 16 & 3 & & 2.91 & $0.30,28.09$ \\
\hline Node $(\mathrm{N})$ stage prior to NAC & & & 0.558 & & \\
\hline Negative & 55 & 6 & & & \\
\hline Positive & 217 & 29 & & 1.29 & $0.54,3.11$ \\
\hline Clinical stage & & & 0.847 & & \\
\hline$\|$ & 185 & 24 & & & \\
\hline III & 86 & 11 & & 0.93 & $0.46,1.91$ \\
\hline Unknown & 1 & & & & \\
\hline Age & & & 0.008 & & \\
\hline$\leq 50$ years at diagnosis & 177 & 17 & & & \\
\hline$>50$ years at diagnosis & 95 & 18 & & 2.49 & $1.28,4.85$ \\
\hline Menopausal status & & & 0.017 & & \\
\hline Premenopausal & 161 & 15 & & & \\
\hline Perimenopausal & 16 & 2 & & 1.42 & $0.32,6.24$ \\
\hline Postmenopausal & 91 & 18 & & 2.74 & $1.38,5.46$ \\
\hline Unknown & 4 & & & & \\
\hline Histology ${ }^{a}$ & & & 0.835 & & \\
\hline Adenocarcinoma, $n$ & 18 & 3 & & & \\
\hline Ductal carcinoma & 207 & 27 & & 1.39 & $0.42,4.62$ \\
\hline Lobular carcinoma & 39 & 4 & & 0.93 & $0.21,4.16$ \\
\hline Other & 8 & 1 & & 1.08 & $0.11,10.42$ \\
\hline Progesterone receptor ${ }^{a}$ & & & 0.199 & & \\
\hline Negative & 76 & 13 & & & \\
\hline Positive & 192 & 21 & & 0.63 & $0.31,1.26$ \\
\hline Unknown & 4 & & & & \\
\hline Tumor grade ${ }^{a}$ & & & 0.14 & & \\
\hline Good & 28 & 2 & & & \\
\hline Moderate & 117 & 16 & & 3.57 & $0.8,15.93$ \\
\hline Poor & 32 & 5 & & 3.52 & $0.66,18.73$ \\
\hline
\end{tabular}

Unknown

95

Chemotherapy regimen

0.89

$\mathrm{ddAC}$

167

$A C-C D$

$A D$

CD

Unknown

Pathologic response 
Table 1 Univariable Cox proportional hazard analysis of relationship between clinical variables and recurrence-free survival (Continued)

\begin{tabular}{|c|c|c|c|c|c|c|}
\hline \multirow[t]{2}{*}{ ypTo/is: } & No & 251 & 34 & 0.29 & & \\
\hline & yes & 21 & 1 & & 0.39 & $0.05,2.88$ \\
\hline \multirow[t]{2}{*}{ ypT < mic: } & No & 221 & 28 & 0.91 & & \\
\hline & yes & 51 & 7 & & 0.95 & $0.42,0.91$ \\
\hline
\end{tabular}

Univariable Cox model for clinical and pathologic parameters of recurrence-free survival. ${ }^{\mathrm{a} D e t e r m i n e d}$ on pre-chemotherapy ultrasound-guided biopsy. ${ }^{\mathrm{b}}$ - $\mathrm{Cl}$ boundary could not be estimated. NAC neoadjuvant chemotherapy, $C l$ confidence interval , (dd)AC (dose-dense) cyclophosphamide and doxorubcin, $C D$ capecitabine and docetaxel, $A D$ doxorubcin and docetaxel, ypTO/isypNO no residual invasive tumor in breast and axilla, ypTO/is no residual invasive tumor in the breast, урT < mic few scattered tumor cells in the breast. Numbers in bold are significant values

analyzed as categorical variables (Table 2) or continuous variables (Table 3). For the categorical predictors, the first mentioned category was taken as a reference and the hazard ratio (HR) compares the subsequent categories to the reference. For the continuous predictors, the $\mathrm{HR}$ represents a change in hazard for one unit change in the predictor.

The clinical and MRI characteristics were first tested for association with the outcome in univariable Cox models. Next, the significant and clinically relevant parameters were analyzed jointly in a multivariable Cox model. When at least one of the analyzed subgroups had no events, the Cox regression with Firth's penalized likelihood was used for the estimation of the hazard ratios. Confidence intervals were then computed using profile likelihood. This technique has been implemented in the $R$ package coxphf.

The optimal cut points and their significance for the continuous variables were estimated using maximally selected rank statistics as implemented in the $\mathrm{R}$ package maxstat. Variables for which the $p$ value was $<0.05$ were considered significant. The final model was built by combining statistical evidence (significant $p$ values) and clinical relevance (age, pathological response). All statistical analyses were performed using $\mathrm{R}$ software (version 3.1.0) or SPSS (version 20).

\section{Results}

Between January 2000 and June 2012428 patients with ER-positive/HER2-negative breast cancer were registered in the NAC breast database of our institute. Of these, 279 patients had response evaluation with MRI (before, during and after), underwent surgery and had no distant metastasis. Seven patients were excluded; four because of a history of breast cancer, two because of technically inadequate MRI, and one patient because she was found to have HER2-positive breast cancer. The majority of the 272 women were premenopausal, had invasive ductal carcinoma, positive nodal stage prior to NAC and tumor stage T2 tumors (Table 1). The median (range) of the measurements of the largest diameter of the initial tumor on MRI was $4.3 \mathrm{~cm}(1.0-11.5)$. The median age at diagnosis was 47 years (range 19-68). The median follow-up time was 41 months (3.4 years).

There were 35 women with an event; 31 women had distant metastases, 2 had additional local/regional recurrence, one only a local/regional recurrence and one patient died without any recurrence reported. There were 20 deaths: 19 breast-cancer-related deaths and 1 death due to another malignancy. The RFS for the study group is shown in Fig. 1.

\section{Univariable Cox model for clinical and pathological parameters}

Among the clinical and pathological parameters, postmenopausal status $(\mathrm{HR}=2.73, p=0.04)$ and age over 50 years $(\mathrm{HR}=2.49, p=0.01)$ were associated with worse RFS (Table 1). pCR, according to any investigated definition, was not associated with improved RFS (Table 1, Fig. 2). Twenty-one patients (7.7 \%) achieved an ypT0/is of the primary tumor after NAC. Only one recurrence was found in this group $(\mathrm{p}=0.29)$. Eleven $(4 \%)$ patients had no residual invasive tumor in the breast or axilla (ypT0/isypN0) after NAC. In this group, no events occurred $(p=0.41)$. Also, a near pCR (a few scattered tumor cells in the breast (ypT $<$ mic)) was observed in 51 patients (seven events), which was not associated with RFS $(p=0.91)$. Kaplan-Meier curves for the pathologic response are shown in Fig. 2.

\section{Univariable Cox model for MRI parameters}

No tumor enhancement (i.e., a radiological complete response) after NAC $(\mathrm{HR}=12.81, p=0.004)$ was significantly associated with superior RFS (Table 2). Forty-four of the 272 patients (16.2 \%) achieved a radiological complete response after NAC as identified on MRI. No events were found in this group. Kaplan-Meier curves for patients with ER-positive/HER2-negative breast cancer show significant difference in RFS between patients with a radiological complete response and those with residual enhancement on MRI (log-rank $p=0.012$; Fig. 3).

Also the largest diameter of the region with washout/ plateau (late) enhancement was associated with RFS on baseline MRI $(\mathrm{HR}=1.017, p=0.027)$, during NAC $(\mathrm{HR}=$ 
Table 2 Univariable Cox proportional hazard analysis of relationship between MRI variables and recurrence-free survival

\begin{tabular}{llllll}
\hline Variable & $\begin{array}{l}\text { Number of } \\
\text { patients }\end{array}$ & Number of & & \multicolumn{2}{l}{ Recurrence free survival } \\
\cline { 5 - 6 } & events & Hazard ratio & $95 \% \mathrm{Cl}$ \\
\hline Lesion morphology baseline MRI & 91 & 10 & 0.612 & 1.29 & $0.71,3.70$ \\
$\quad$ Mass unifocal & 96 & 12 & 1.60 & $0.57,3.01$ \\
Mass multifocal & 77 & 13 & 4.38 & $0.03,41.66$
\end{tabular}

Pattern of reduction at MRI after NAC

0.029

No change

Shrinking mass

Diffuse decrease

Small foci

No enhancement

Dynamic curve type after NAC

No enhancement

Continuous

Plateau

Washout

Radiological complete response

Yes

No

RECIST evaluation MRI initial after NAC - baseline

No enhancement after NAC

Part Rem (LD initial $\downarrow \geq 30 \%)$

NR (LD initial $\downarrow<30 \%)$

RECIST evaluation MRI initial after NAC - during

No enhancement during and after NAC

No enhancement after NAC

Part Rem (LD initial $\downarrow \geq 30 \%)$

NR (LD initial $\downarrow<30 \%)$

RECIST evaluation MRI late after NAC - baseline

No washout/plateau baseline and after NAC

No washout/plateau after NAC

Part Rem (LD late $\downarrow \geq 30 \%)$

NR (LD late $\downarrow<30 \%)$

RECIST evaluation MRI late after NAC - during

No plateau/washout during and after NAC

No plateau/washout after NAC

Part Rem (LD late $\downarrow \geq 30 \%)$

NR (LD late $\downarrow<30 \%$ )
23

96

56

53

44

44

44

89

82

57

4

44

228

44

44

154

74

10

10

36

82

144

\section{8}

136

93

35

61

84

58

69

\section{4}

10

10

11

0

\section{0}

16

5

14

0

35

0

0

0

11

24

0

Univariable Cox proportional hazard analysis of magnetic resonance imaging (MRI) variables with recurrence-free survival. ${ }^{\mathrm{a}} \mathrm{Cl}$ boundary could not be estimated. $\mathrm{Cl}$ confidence interval, $L D$ largest diameter, Part Rem partial remission, initial enhancement $90 \mathrm{~s}$, late washout/plateau enhancement $450 \mathrm{~s}$, MRI magnetic resonance imaging, NAC neoadjuvant chemotherapy, NR non responder, RECIST response evaluation criteria in solid tumors. Arrow ( $\downarrow$ ) indicates decrease. Numbers in bold are significant values
0.008

$\begin{array}{ll}0.56 & 0.19,1.89 \\ 0.78 & 0.27,2.64 \\ 0.95 & 0.34,3.19 \\ 0.06 & 0,0.53\end{array}$

$\begin{array}{ll}13.54 & 1.83,1728.03 \\ 7.46 & 0.84,980.87 \\ 17.59 & 2.35,2248.46\end{array}$

0.004

0.009

$\begin{array}{ll}11.63 & -^{\mathrm{a}}, 1477.68 \\ 16.53 & 2.17,2119.55\end{array}$

0.037

$\begin{array}{ll}0.44 & 0,80.78 \\ 3.99 & 0.52,513.45 \\ 4.95 & 0.68,629.57\end{array}$

0.05

$\begin{array}{ll}3.08 & 0.41,394.53 \\ 3.54 & 0.46,455.8 \\ 11.57 & 1.31,1525.99\end{array}$

0.46

\begin{tabular}{lc}
1.17 & $0.43,3.17$ \\
1.65 & $0.59,4.64$ \\
2.08 & $0.74,5.87$ \\
\hline l boundary could not be estimated. Cl \\
ment $450 \mathrm{~s}, \mathrm{MRI}$ magnetic resonance \\
indicates decrease. Numbers in bold
\end{tabular}


Table 3 Univariable Cox proportional hazard analysis of relationship between continuous MRI variables and recurrence-free survival

\begin{tabular}{|c|c|c|c|c|}
\hline \multirow[b]{2}{*}{ MRI variable } & \multirow[b]{2}{*}{ Median } & \multicolumn{3}{|c|}{ Recurrence-free survival } \\
\hline & & $P$ value & Hazard ratio & $95 \% \mathrm{Cl}$ \\
\hline \multicolumn{5}{|l|}{ Baseline (before NAC) } \\
\hline Largest diameter MIP/initial enhancement (90 s) & $43 \mathrm{~mm}$ & 0.238 & 1,009 & $0.994,1.024$ \\
\hline Largest diameter plateau/washout enhancement (450 s) & 33 mm & 0.027 & 1,017 & $1.002,1.033$ \\
\hline Initial enhancement (90 s) \% & $152 \%$ & 0.326 & 0.997 & $0.99,1.003$ \\
\hline Late enhancement (450 s) \% & $-13 \%$ & 0.947 & 0.999 & $0.967,1.032$ \\
\hline \multicolumn{5}{|l|}{ During NAC } \\
\hline Largest diameter MIP/initial enhancement (90s) & $30 \mathrm{~mm}$ & 0.155 & 1,011 & $0.996,1.026$ \\
\hline Largest diameter plateau/washout enhancement (450 s) & $17 \mathrm{~mm}$ & 0.006 & 1,024 & $1.007,1.041$ \\
\hline Initial enhancement \% & $135 \%$ & 0.993 & 1.00 & $0.995,1.005$ \\
\hline Late enhancement \% & $-4 \%$ & 0.600 & 0.995 & $0.975,1.015$ \\
\hline \multicolumn{5}{|l|}{ After NAC } \\
\hline Largest diameter MIP/initial enhancement $(90 \mathrm{~s})^{a}$ & $22 \mathrm{~mm}$ & 0.140 & 1.01 & $1.00,1.03$ \\
\hline Largest diameter plateau/washout enhancement (450 s) & $0 \mathrm{~mm}$ & 0.003 & 1.03 & $1.01,1.051$ \\
\hline Initial enhancement \% & $100 \%$ & 0.057 & 1,005 & $1.00,1.011$ \\
\hline Late enhancement \% & $9 \%$ & 0.815 & 0.998 & $0.983,1.014$ \\
\hline \multicolumn{5}{|l|}{ Percent change after NAC - baseline NAC \% } \\
\hline Largest diameter MIP/initial enhancement $(90 \mathrm{~s})^{\mathrm{b}}$ & $-40 \%$ & 0.280 & 1.01 & $0.99,1.02$ \\
\hline Largest diameter plateau/washout enhancement $(450 \mathrm{~s})^{c}$ & $-100 \%$ & 0.021 & 1,013 & $1.002,1.024$ \\
\hline \multicolumn{5}{|l|}{ Percent change after NAC - during NAC \% } \\
\hline Largest diameter MIP/initial enhancement (90 s) mm b & $-30 \%$ & 0.290 & 1.01 & $0.99,1.02$ \\
\hline Largest diameter plateau/washout enhancement $(450 \mathrm{~s})^{c}$ & $-61 \%$ & 0.066 & 1,008 & $0.999,1.017$ \\
\hline
\end{tabular}

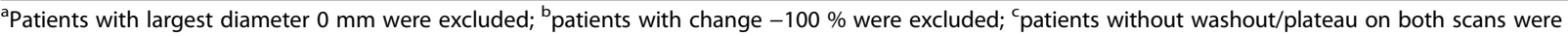
excluded. MRI magnetic resonance imaging, $\mathrm{Cl}$ confidence interval, NAC neoadjuvant chemotherapy, MIP maximum intensity projection. Numbers in bold are significant

1.024, $p=0.006)$ and after NAC $(\mathrm{HR}=1.03, p=0.003)$ (Table 3). The most significant cut off for the largest diameter of washout/plateau enhancement after NAC was estimated for the value $22 \mathrm{~mm}$. Log-rank test $p$ value $<0.001$ (Fig. 4). In addition, the percent change in the largest diameter of the region with washout/plateau enhancement between baseline and after NAC $(\mathrm{HR}=1.013, p=0.021)$ was associated with RFS (Table 3).

\section{Multivariable analysis}

In the multivariable analysis we fitted a Cox model including radiological complete response after NAC, the largest diameter of washout/plateau on MRI after NAC, the patient's age and pathological response (yp T< $\mathrm{mic})$.The first three predictors remained statistically significant with HR of $14.11(1.8-1818, p=0.006), 1.02$ $(1.00-1.04, p=0.036)$ and $2.55(1.3-5.02, p=0.007)$, respectively. Pathological response did not remain significant; HR $=2.12(0.86-4.64, p=0.096)$.

\section{Discussion}

In a series of 272 consecutive patients with luminal (ERpositive/HER2-negative) breast cancer, radiological complete remission assessed on MRI after NAC was associated with significantly improved RFS after NAC. All of the 44 patients $(16 \%)$ with radiological complete response remained free of disease during follow up.

This finding may be of clinical importance. Luminal breast cancer is the most common breast cancer and represents approximately $2 / 3$ of all cases. Patients with luminal tumors only rarely achieve pCR. In this study, only $8 \%(21 / 272)$ achieved pCR in the breast and even fewer patients $(4 \%(11 / 272))$, achieved $\mathrm{pCR}$ in the breast and axilla. In our study neither pCR (i.e., ypT0is or ypT0isN0) nor near-pCR was predictive of improved RFS. These findings are in accordance with previously published work [17]. Also other studies showed that pCR is not a suitable surrogate endpoint for patients with ER-positive/HER2negative grade 1 or 2 (luminal A) breast cancer $[35,36]$.

We investigated the potential of MRI to predict recurrence-free survival. MRI after completion of chemotherapy was found to be of particular prognostic value in the current study. Apparently, the lack of enhancement on MRI, which provides information about functional properties of the tumor, is associated with prognosis in slowly proliferating tumors, but $\mathrm{pCR}$ is not. 


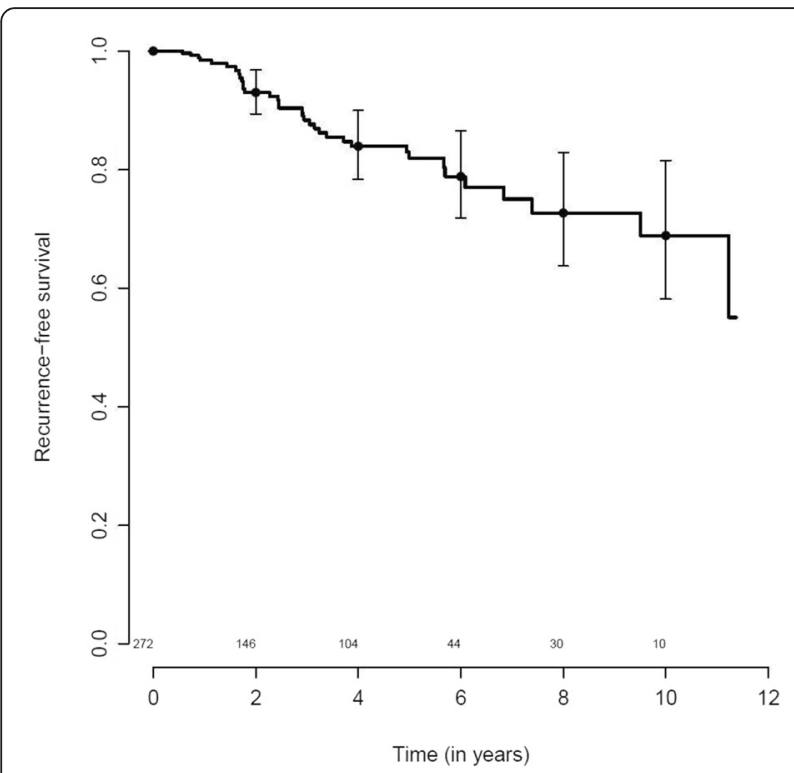

Fig. 1 Recurrence-free survival among 272 patients with estrogen receptor (ER)-positive/human epidermal growth factor receptor 2 (HER2)-negative breast cancer after neoadjuvant chemotherapy (solid line), and the $95 \%$ confidence interval. Numbers of patients at risk are shown above the $x$-axis

Many studies have evaluated the role of MRI after NAC as a diagnostic tool to serve as a surrogate for final pathology [37-39]. The majority of studies have focused on the correlation between tumor size as assessed by MRI and that identified on pathology assessment to validate MRI as a tool to detect residual disease and to guide surgical planning. In terms of tumor size, MRI may underestimate or overestimate compared to pathology assessment, resulting in false-negative and falsepositive results $[40,41]$. Other studies have shown that a complete response after neoadjuvant chemotherapy identified on MRI is associated with the presence of residual tumor pathology assessment in 26-56 \% of cases $[26,42]$. More recent studies have indicated that the accuracy of MRI in estimating tumor size after neoadjuvant chemotherapy varies with breast cancer subtype and tumor morphology [22, 24, 39, 43]. The best accuracy is achieved in HER2-positive and triple-negative tumors [22, 24, 39]. We found that a radiological complete response in ER-positive breast cancer is associated with an excellent prognosis. However in $36 \%(16 / 44)$ of these cases, there was (microscopic) residual tumor on the final pathology assessment.

We used a very strict definition of a radiological complete response in which even small enhancing foci in the original tumor bed are considered as residual tumor. Especially in diffuse tumors (non-mass enhancement) that disintegrate into (very) small foci the radiological assessment can be challenging and in clinical practice small enhancing foci may occasionally be interpreted incorrectly as a radiological complete response. We have observed such interpretation discrepancies between the retrospective dedicated review of our study and the clinical routine MRI assessment. For future validation studies it will be important to maintain the strict definition of radiological complete response.

The policy of changing the chemotherapy regimen in the case of an unfavorable MRI response during NAC could have led to an increase in the (radiological) complete remission rate in our study. This was certainly the objective of the policy, but whether this really succeeded needs to be further studied in controlled trials. We assumed that a larger reduction in tumor size on MRI could correlate with a smaller volume of residual tumor, but it could also serve as a measure of chemotherapy sensitivity. The latter could be critically important for the likelihood that micro-metastatic disease has been eradicated or reduced, which is the primary objective of NAC. The differences between radiological complete remission (CR) and $\mathrm{pCR}$ in this respect, include the more frequent occurrence of radiological CR in this type of tumor and perhaps the higher likelihood of radiological CR in tumor subtypes that tend to recur less often or later than others. Although a detailed subgroup analysis could not be performed due to the limited number of patients, there was no indication that the association between radiological CR and RFS was different for different chemotherapy regimens or between patients who did and those who did not cross over to a different chemotherapy regimen (Table 1).

The value of MRI with or without prognostic markers such as those derived from pathology assessment is yet unclear when it comes to predicting disease-free survival of patients with ER-positive/HER2-negative breast cancer. A few studies have investigated the predictive role of MRI in breast cancer survival after NAC without using a distinction in subgroups [44-46]. In a relatively small study group of 58 patients with a short median follow up of 33 months, Partridge et al. showed that initial MRI volume before NAC, and final change in MRI volume were significant predictors of RFS [44]. Yi et al. evaluated 158 breast cancer patients with MRI before and after NAC. They concluded that a smaller reduction in tumor volume and a smaller reduction in washout component, assessed with computer-aided evaluation, were associated with worse RFS [45].

Jafri et al. evaluated the optimal threshold for measuring functional tumor volume in 64 patients. They concluded that functional tumor volume is able to predict RFS and could be used as a biomarker [46]. These three studies did not report how many patients achieved radiological or clinical complete response on MRI, nor did they analyze breast cancer subgroups. 


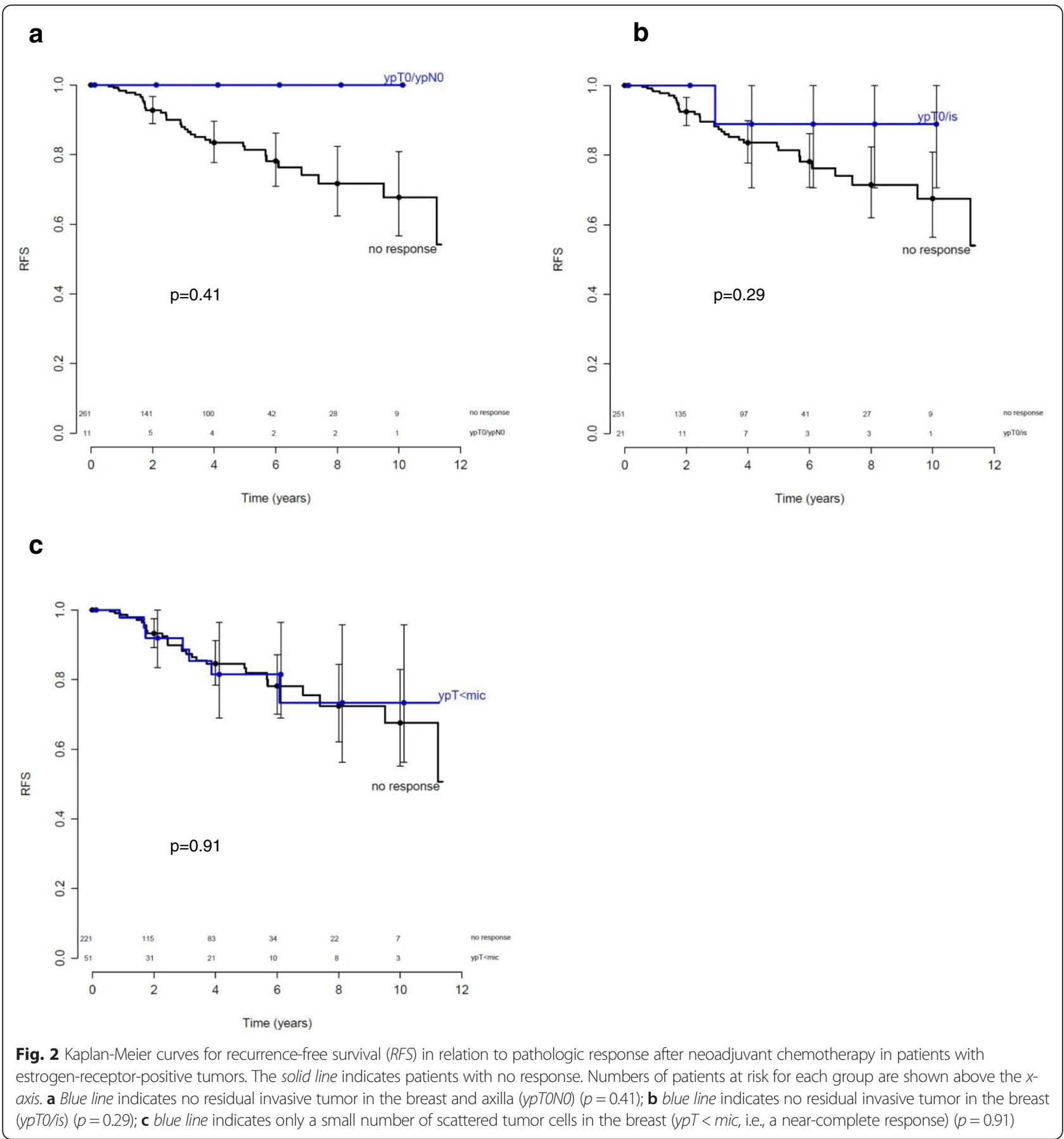

A more recent published study (ACRIN 6657) noted that functional tumor volume (tumor volume percent enhancement $>70 \%$ ) after NAC is a strong predictor of RFS in breast cancer [25]. Their Kaplan-Meier analyses performed by subtype suggest that the ability of functional tumor volume to discriminate differences differs per breast cancer subtype. After NAC, a greater RFS separation was found in 78 ER-positive/HER2-negative and 41 HER2-positive breast cancers than in the whole group. Instead of volumetric measurements, we assessed the largest diameter at initial (MIP, $90 \mathrm{~s}$ ) and late (washout/plateau) enhancement on MRI. In accordance with Yi et al. we found that the largest diameter of washout/ plateau and the change in this diameter are significantly associated with RFS in our subset of ER-positive breast cancer. However, in daily clinical practice a radiological complete response is a more straightforward and potentially a more reproducible measure to identify patients 


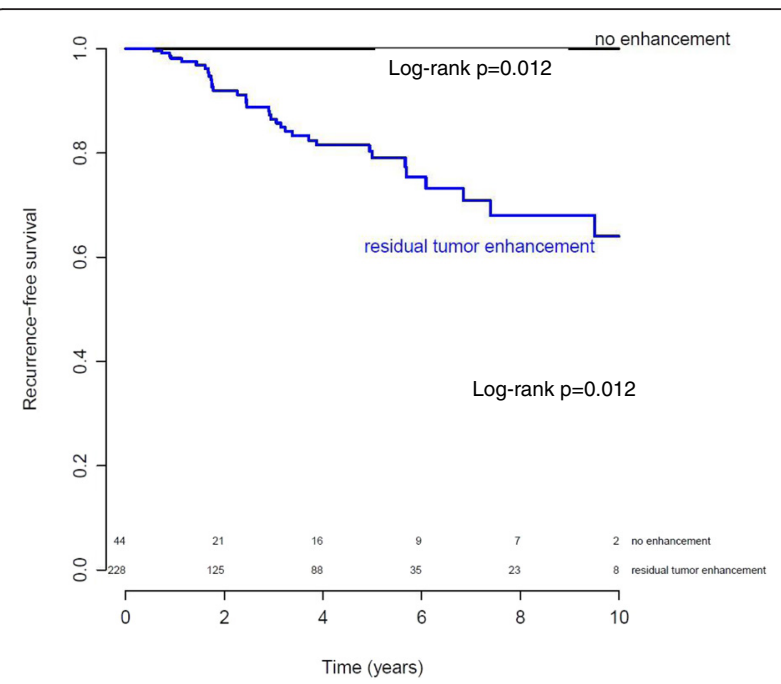

Fig. 3 Kaplan-Meier curves for recurrence-free survival (RFS) of patients with estrogen-receptor-positive tumors based on radiological complete response (black line no enhancement) and those with residual enhancement (blue line) identified on magnetic resonance imaging after neoadjuvant chemotherapy. Log-rank test $p=0.012$. Numbers of patients at risk in each group are shown above the $x$-axis

with ER-positive/HER2-negative breast cancer who have a good prognosis. On the other hand, in patients with residual enhancement on MRI, and who thus may have a less favorable prognosis, the largest diameter of washout/plateau enhancement may be used to decide if

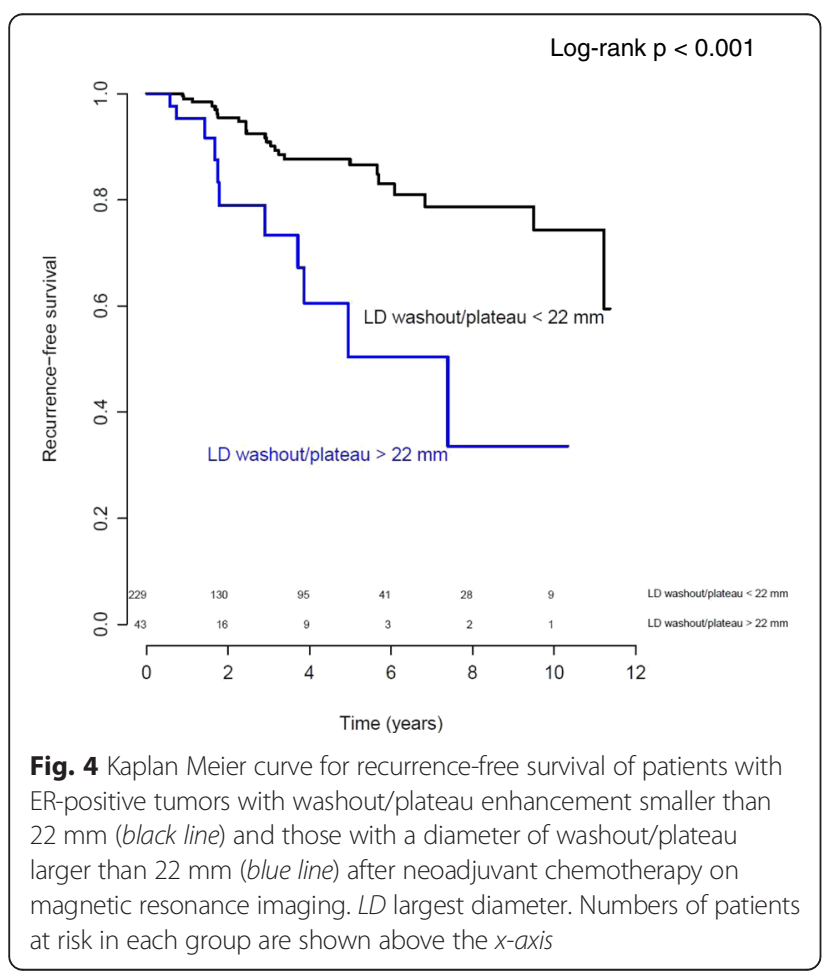

additional chemotherapy is required. For this study population the most significant cut off was estimated for a largest diameter of $22 \mathrm{~mm}$. However, before we can actually use this value we need to validate this in a larger study group, preferably with longer follow up.

Our study has some limitations. These involve potential suboptimal selection of groups and differences in chemotherapy regimens. The study ran for an extensive period of time (2000-2012). During this time, the $1.5 \mathrm{~T}$ MRI scanner was replaced by a $3 \mathrm{~T}$ scanner, and the MRI scan protocol was amended to standard clinical care. Care was taken to align the MRI protocols over time between $1.5 \mathrm{~T}$ and $3 \mathrm{~T}$ as much as possible, but minor differences could not be avoided in voxel size and FOV. During the study period the temporal resolution and methods used to analyze the images remained unchanged. Although we have no indication that this is the case, one can never be certain that small differences in scan protocols may affect the results in some way. This is a limitation that is difficult to avoid in longer-running radiological studies such as those presented here, given the rapid developments in MRI technology that inevitably find their way into daily clinical practice. Nonetheless, despite these differences, we were still able to demonstrate significant associations. In addition, the MRI measurements were performed interactively on the basis of automatically calculated color overlay images by different radiologists. Even though the measurements (largest diameter, ROI placement for relative enhancement percentage) were carried out carefully by dedicated breast radiologists and according to protocol this manual procedure is to a certain extent subjective and can lead to potential bias. Although more recent methods of volumetric assessment may further reduce subjectivity, it is difficult to avoid it altogether due to empirical adjustments of parameters such as percent-enhancement thresholds and placement of the region of interest [25].

Although the total study group is relatively large, only 35 recurrences occurred during a follow-up time that is relatively brief for ER-positive/HER2-negative (luminal) tumors. This resulted in wide confidence intervals for the hazard ratios. In this study the tumor grade determined on the biopsy was known in only 177 (65\%) patients. As a result we were not able to allow additional stratification in luminal A and luminal B tumors. Ideally, subtyping would also have been based on gene expression rather than on immunohistochemical assessment, and the median follow up would have been longer, with more recurrences available for analysis. On the other hand, the predictive effect of a radiologic complete response may be especially clear in the first 5 years after NAC. The Oxford overview has shown that chemotherapy prevents recurrences within the first 5 years, while the preventive effect of endocrine treatment, which is at 
least as important in luminal tumors, extends beyond 10 years [47]. As a result, effective chemotherapy may prevent early relapse, seen after limited follow up, when the endocrine treatment effect is not yet dominant.

Another limitation is that different dedicated breast radiologists in a single institution using strict criteria assessed the results. As a result, we were not able to evaluate inter-observer or intra-observer variability. Further exploration with longer follow up and an external validation cohort will be useful to validate our results.

\section{Conclusions}

In conclusion, radiologic complete response on MRI after NAC in patients with ER-positive/HER2-negative tumors is associated with an excellent outcome. In the case of residual enhancement on MRI after NAC, the largest diameter of late enhancement may be helpful to identify patients who may need additional treatment.

\begin{abstract}
Abbreviations
AC, doxorubicin and cyclophosphamide; AD; doxorubcin and docetaxel; $C R$, complete remission; ddAC, (dose-dense) cyclophosphamide and doxorubicin; ER, estrogen receptor; HER2, human epidermal growth factor receptor 2; HR, hazard ratio; IHC, immunohistochemical assessment; MIP, maximum intensity projection; MRI, magnetic resonance imaging; NAC, neoadjuvant chemotherapy; $\mathrm{pCR}$, pathological complete remission; PR, progesterone receptor; RFS, recurrence-free survival; ypTO/is, no residual invasive tumor in the breast; ypT0/ isypNO, no residual invasive tumor in breast and axilla; ypT < mic, few scattered tumor cells in the breast
\end{abstract}

\section{Acknowledgments}

We thank Anita Paape, Gonneke Winter, Petra de Koekkoek and Annemarie Fioole of the radiology department and Nicola Russell of the radiotherapy department for their dedication and contributions.

\section{Funding}

This study was in part performed within the framework of CTMM, the center for Translational Molecular Medicine (www.ctmm.nl); project Breast CARE (grant 030-104).

\section{Authors' contributions}

Study design and concept: KG, SR, CL and LR. Data acquisition: CL, LR, KP and JW. Data analysis and interpretation: CL, SR, KS and KG. Statistical analysis: KS, $K G, C L$ and SR. Manuscript writing: CL, SR and KG. Manuscript editing: $C L, L R, K P$, JW, SR, MVP, KS and KG. Manuscript review: CL, LR, KP, JW, SR, MVP, KS and KG. All authors have read and approved the final version of the manuscript.

\section{Competing interests}

The authors declare that they have no competing interests.

\section{Ethics approval and consent to participate}

The Medical Research Ethics Committee (MREC) of the Netherlands Cancer Institute approved the study.

\section{Author details}

${ }^{1}$ Division of Diagnostic Oncology (Department of Radiology), The Netherlands Cancer Institute - Antoni van Leeuwenhoek Hospital, Plesmanlaan 121, Amsterdam 1066 CX, The Netherlands. '2Division of Medical Oncology, The Netherlands Cancer Institute - Antoni van Leeuwenhoek Hospital, Plesmanlaan 121, Amsterdam 1066 CX, The Netherlands. ${ }^{3}$ Division of Diagnostic Oncology (Department of Pathology) and Division of Molecular Pathology, The Netherlands Cancer Institute - Antoni van Leeuwenhoek Hospital, Plesmanlaan 121, Amsterdam 1066 CX, The Netherlands. ${ }^{4}$ Division of Surgical oncology, The Netherlands Cancer Institute - Antoni van Leeuwenhoek Hospital, Plesmanlaan 121, Amsterdam 1066 CX. The Netherlands. ${ }^{5}$ Department of Biostatistics, The Netherlands Cancer Institute -
Antoni van Leeuwenhoek Hospital, Plesmanlaan 121, Amsterdam 1066 CX, The Netherlands. ${ }^{6}$ Department of Radiology and the Image Science Institute, University Medical Center Utrecht, Utrecht, The Netherlands.

Received: 2 December 2015 Accepted: 25 July 2016

Published online: 05 August 2016

\section{References}

1. Wolmark N, Wang J, Mamounas E, Bryant J, Fisher B. Preoperative chemotherapy in patients with operable breast cancer: nine-year results from National Surgical Adjuvant Breast and Bowel Project B-18. J Natl Cancer Inst Monogr. 2001;30:96-102.

2. Rastogi P, Anderson SJ, Bear HD, Geyer CE, Kahlenberg MS, Robidoux A, Margolese RG, Hoehn JL, Vogel VG, Dakhil SR, et al. Preoperative chemotherapy: updates of National Surgical Adjuvant Breast and Bowel Project Protocols B-18 and B-27. J Clin Oncol. 2008;26(5):778-85.

3. Fisher B, Brown A, Mamounas E, Wieand S, Robidoux A, Margolese RG, Cruz Jr AB, Fisher ER, Wickerham DL, Wolmark N, et al. Effect of preoperative chemotherapy on local-regional disease in women with operable breast cancer: findings from National Surgical Adjuvant Breast and Bowel Project B-18. J Clin Oncol. 1997;15(7):2483-93.

4. Fisher ER, Wang J, Bryant J, Fisher B, Mamounas E, Wolmark N. Pathobiology of preoperative chemotherapy: findings from the National Surgical Adjuvant Breast and Bowel (NSABP) protocol B-18. Cancer. 2002;95(4):681-95.

5. Denley H, Pinder SE, Elston CW, Lee AH, Ellis IO. Preoperative assessment of prognostic factors in breast cancer. J Clin Pathol. 2001;54(1):20-4.

6. Rakha EA, El Sayed ME, Green AR, Paish EC, Powe DG, Gee J, Nicholson RI, Lee AH, Robertson JF, Ellis IO. Biologic and clinical characteristics of breast cancer with single hormone receptor positive phenotype. J Clin Oncol. 2007;25(30):4772-8.

7. Chuthapisith S, Eremin JM, Eremin O. Predicting response to neoadjuvant chemotherapy in breast cancer: molecular imaging, systemic biomarkers and the cancer metabolome (Review). Oncol Rep. 2008;20(4):699-703.

8. Desmedt C, Haibe-Kains B, Wirapati P, Buyse M, Larsimont D, Bontempi G, Delorenzi M, Piccart M, Sotiriou C. Biological processes associated with breast cancer clinical outcome depend on the molecular subtypes. Clin Cancer Res. 2008;14(16):5158-65.

9. Sanchez-Munoz A, Garcia-Tapiador AM, Martinez-Ortega E, Duenas-Garcia R, Jaen-Morago A, Ortega-Granados AL, Fernandez-Navarro M, Torre-Cabrera C, Duenas B, Rueda Al, et al. Tumour molecular subtyping according to hormone receptors and HER2 status defines different pathological complete response to neoadjuvant chemotherapy in patients with locally advanced breast cancer. Clin Transl Oncol. 2008;10(10):646-53.

10. de Ronde JJ, Hannemann J, Halfwerk H, Mulder L, Straver ME, Vrancken Peeters MJ, Wesseling J, van de Vijver M, Wessels LF, Rodenhuis S. Concordance of clinical and molecular breast cancer subtyping in the context of preoperative chemotherapy response. Breast Cancer Res Treat. 2010;119(1):119-26.

11. Nicholson RI, Johnston SR. Endocrine therapy-current benefits and limitations. Breast Cancer Res Treat. 2005;93 Suppl 1:S3-S10.

12. Tokunaga E, Oki E, Nishida K, Koga T, Egashira A, Morita M, Kakeji Y, Maehara Y. Trastuzumab and breast cancer: developments and current status. Int J Clin Oncol. 2006;11(3):199-208.

13. Chollet P, Amat S, Cure H, de Latour M, Le Bouedec G, Mouret-Reynier MA, Ferriere JP, Achard JL, Dauplat J, Penault-Llorca F. Prognostic significance of a complete pathological response after induction chemotherapy in operable breast cancer. Br J Cancer. 2002;86(7):1041-6.

14. Sataloff DM, Mason BA, Prestipino AJ, Seinige UL, Lieber CP, Baloch Z. Pathologic response to induction chemotherapy in locally advanced carcinoma of the breast: a determinant of outcome. J Am Coll Surg. 1995; 180(3):297-306.

15. Fisher B, Bryant J, Wolmark N, Mamounas E, Brown A, Fisher ER, Wickerham DL, Begovic M, DeCillis A, Robidoux A, et al. Effect of preoperative chemotherapy on the outcome of women with operable breast cancer. J Clin Oncol. 1998;16(8):2672-85.

16. van der Hage JA, van de Velde CJ, Julien JP, Tubiana-Hulin M, Vandervelden C, Duchateau L. Preoperative chemotherapy in primary operable breast cancer: results from the European Organization for Research and Treatment of Cancer trial 10902. J Clin Oncol. 2001;19(22):4224-37.

17. von Minckwitz G, Untch M, Blohmer JU, Costa SD, Eidtmann H, Fasching PA Gerber B, Eiermann W, Hilfrich J, Huober J, et al. Definition and impact of 
pathologic complete response on prognosis after neoadjuvant chemotherapy in various intrinsic breast cancer subtypes. J Clin Oncol. 2012; 30(15):1796-804

18. Abraham DC, Jones RC, Jones SE, Cheek JH, Peters GN, Knox SM, Grant MD, Hampe DW, Savino DA, Harms SE. Evaluation of neoadjuvant chemotherapeutic response of locally advanced breast cancer by magnetic resonance imaging. Cancer. 1996;78(1):91-100.

19. Balu-Maestro C, Chapellier C, Bleuse A, Chanalet I, Chauvel C, Largillier R. Imaging in evaluation of response to neoadjuvant breast cancer treatment benefits of MRI. Breast Cancer Res Treat. 2002;72(2):145-52.

20. Bodini M, Berruti A, Bottini A, Allevi G, Fiorentino C, Brizzi MP, Bersiga A Generali D, Volpi D, Marini U, et al. Magnetic resonance imaging in comparison to clinical palpation in assessing the response of breast cancer to epirubicin primary chemotherapy. Breast Cancer Res Treat. 2004;85(3):211-8.

21. Yeh E, Slanetz P, Kopans DB, Rafferty E, Georgian-Smith D, Moy L, Halpern E, Moore R, Kuter I, Taghian A. Prospective comparison of mammography, sonography, and MRI in patients undergoing neoadjuvant chemotherapy for palpable breast cancer. AJR Am J Roentgenol. 2005;184(3):868-77.

22. Hayashi Y, Takei H, Nozu S, Tochigi Y, Ichikawa A, Kobayashi N, Kurosumi M, Inoue K, Yoshida T, Nagai SE, et al. Analysis of complete response by MRI following neoadjuvant chemotherapy predicts pathological tumor responses differently for molecular subtypes of breast cancer. Oncol Lett. 2013;5(1):83-9.

23. Chen JH, Feig BA, Hsiang DJ, Butler JA, Mehta RS, Bahri S, Nalcioglu O, Su MY. Impact of MRI-evaluated neoadjuvant chemotherapy response on change of surgical recommendation in breast cancer. Ann Surg. 2009;249(3): 448-54.

24. McGuire KP, Toro-Burguete J, Dang H, Young J, Soran A, Zuley M, Bhargava R, Bonaventura M, Johnson R, Ahrendt G. MRI staging after neoadjuvant chemotherapy for breast cancer: does tumor biology affect accuracy? Ann Surg Oncol. 2011;18(11):3149-54.

25. Hylton NM, Gatsonis CA, Rosen MA, Lehman CD, Newitt DC, Partridge SC, Bernreuter WK, Pisano ED, Morris EA, Weatherall PT, et al. Neoadjuvant chemotherapy for breast cancer: functional tumor volume by MR imaging predicts recurrence-free survival-results from the ACRIN 6657/CALGB 150007 I-SPY 1 TRIAL. Radiology. 2015;279(1):44-55.

26. Straver ME, Rutgers EJ, Rodenhuis S, Linn SC, Loo CE, Wesseling J, Russell NS, Oldenburg HS, Antonini N, Vrancken Peeters MT. The relevance of breast cancer subtypes in the outcome of neoadjuvant chemotherapy. Ann Surg Oncol. 2010:Apr 6. [Epub ahead of print].

27. Hannemann J, Oosterkamp HM, Bosch CA, Velds A, Wessels LF, Loo C, Rutgers EJ, Rodenhuis S, van de Vijver MJ. Changes in gene expression associated with response to neoadjuvant chemotherapy in breast cancer. J Clin Oncol. 2005;23(15):3331-42

28. Loo CE, Teertstra HJ, Rodenhuis S, van de Vijver MJ, Hannemann J, Muller SH, Peeters MJ, Gilhuijs KG. Dynamic contrast-enhanced MRI for prediction of breast cancer response to neoadjuvant chemotherapy: initial results. AJR Am J Roentgenol. 2008;191(5):1331-8.

29. Gilhuiij KG, Deurloo EE, Muller SH, Peterse JL, Schultze Kool L. Breast MR imaging in women at increased lifetime risk of breast cancer: clinical system for computerized assessment of breast lesions initial results. Radiology. 2002;225(3):907-16.

30. Kuhl CK, Mielcareck P, Klaschik S, Leutner C, Wardelmann E, Gieseke J, Schild $\mathrm{HH}$. Dynamic breast MR imaging: are signal intensity time course data useful for differential diagnosis of enhancing lesions? Radiology. 1999;211(1):101-10.

31. American College of Radiology. Breast Imaging and Reporting Data System (BI-RADS). Reston: American College of Radiology; 2003.

32. Bear HD, Anderson S, Brown A, Smith R, Mamounas EP, Fisher B, Margolese $\mathrm{R}$, Theoret $\mathrm{H}$, Soran $\mathrm{A}$, Wickerham $\mathrm{DL}$, et al. The effect on tumor response of adding sequential preoperative docetaxel to preoperative doxorubicin and cyclophosphamide: preliminary results from National Surgical Adjuvant Breast and Bowel Project Protocol B-27. J Clin Oncol. 2003;21(22):4165-74.

33. Green MC, Buzdar AU, Smith T, Ibrahim NK, Valero V, Rosales MF, Cristofanilli M, Booser DJ, Pusztai L, Rivera E, et al. Weekly paclitaxel improves pathologic complete remission in operable breast cancer when compared with paclitaxel once every 3 weeks. J Clin Oncol. 2005;23(25):5983-92.

34. Hudis CA, Barlow WE, Costantino JP, Gray RJ, Pritchard KI, Chapman JA, Sparano JA, Hunsberger S, Enos RA, Gelber RD, et al. Proposal for standardized definitions for efficacy end points in adjuvant breast cancer trials: the STEEP system. J Clin Oncol. 2007;25(15):2127-32.
35. Lips EH, Mulder L, de Ronde JJ, Mandjes IA, Koolen BB, Wessels LF, Rodenhuis S, Wesseling J. Breast cancer subtyping by immunohistochemistry and histological grade outperforms breast cancer intrinsic subtypes in predicting neoadjuvant chemotherapy response. Breast Cancer Res Treat. 2013;140(1):63-71.

36. Lips EH, Mulder L, de Ronde JJ, Mandjes IA, Vincent A, Vrancken Peeters MT, Nederlof PM, Wesseling J, Rodenhuis S. Neoadjuvant chemotherapy in ER+ HER2- breast cancer: response prediction based on immunohistochemical and molecular characteristics. Breast Cancer Res Treat. 2012;131(3):827-36.

37. Rosen EL, Blackwell KL, Baker JA, Soo MS, Bentley RC, Yu D, Samulski TV, Dewhirst MW. Accuracy of MRI in the detection of residual breast cancer after neoadjuvant chemotherapy. AJR Am J Roentgenol. 2003;181(5):1275-82.

38. Warren RM, Bobrow LG, Earl HM, Britton PD, Gopalan D, Purushotham AD, Wishart GC, Benson JR, Hollingworth W. Can breast MRI help in the management of women with breast cancer treated by neoadjuvant chemotherapy? Br J Cancer. 2004;90(7):1349-60.

39. De Los Santos JF, Cantor A, Amos KD, Forero A, Golshan M, Horton JK, Hudis CA, Hylton NM, McGuire K, Meric-Bernstam F, et al. Magnetic resonance imaging as a predictor of pathologic response in patients treated with neoadjuvant systemic treatment for operable breast cancer. Translational Breast Cancer Research Consortium trial 017. Cancer. 2013; 119(10):1776-83.

40. Kwong MS, Chung GG, Horvath LJ, Ward BA, Hsu AD, Carter D, Tavassoli F, Haffty B, Burtness BA. Postchemotherapy MRI overestimates residual disease compared with histopathology in responders to neoadjuvant therapy for locally advanced breast cancer. Cancer J. 2006;12(3):212-21.

41. Rieber A, Zeitler H, Rosenthal H, Gorich J, Kreienberg R, Brambs HJ, Tomczak $\mathrm{R}$. MRI of breast cancer: influence of chemotherapy on sensitivity. $\mathrm{Br}$ J Radiol. 1997;70(833):452-8

42. Chen JH, Feig B, Agrawal G, Yu H, Carpenter PM, Mehta RS, Nalcioglu O, Su MY. MRI evaluation of pathologically complete response and residual tumors in breast cancer after neoadjuvant chemotherapy. Cancer. 2008; 112(1):17-26

43. Straver ME, Loo CE, Rutgers EJ, Oldenburg HS, Wesseling J, Vrancken Peeters MJ, Gilhuijs KG. MRI-model to guide the surgical treatment in breast cancer patients after neoadjuvant chemotherapy. Ann Surg. 2010;251(4):701-7.

44. Partridge SC, Gibbs JE, Lu Y, Esserman LJ, Tripathy D, Wolverton DS, Rugo HS, Hwang ES, Ewing CA, Hylton NM. MRI measurements of breast tumor volume predict response to neoadjuvant chemotherapy and recurrence-free survival. AJR Am J Roentgenol. 2005;184(6):1774-81.

45. Yi A, Cho N, Im SA, Chang JM, Kim SJ, Moon HG, Han W, Park IA, Noh DY, Moon WK. Survival outcomes of breast cancer patients who receive neoadjuvant chemotherapy: association with dynamic contrast-enhanced MR imaging with computer-aided evaluation. Radiology. 2013;268(3):662-72

46. Jafri NF, Newitt DC, Kornak J, Esserman LJ, Joe BN, Hylton NM. Optimized breast MRI functional tumor volume as a biomarker of recurrence-free survival following neoadjuvant chemotherapy. J Magn Reson Imaging. 2014; 40(2):476-82.

47. Early Breast Cancer Trialists' Collaborative Group (EBCTCG). Effects of chemotherapy and hormonal therapy for early breast cancer on recurrence and 15-year survival: an overview of the randomised trials. Lancet. 2005; 365(9472):1687-1717.

\section{Submit your next manuscript to BioMed Central and we will help you at every step:}

- We accept pre-submission inquiries

- Our selector tool helps you to find the most relevant journal

- We provide round the clock customer support

- Convenient online submission

- Thorough peer review

- Inclusion in PubMed and all major indexing services

- Maximum visibility for your research

Submit your manuscript at www.biomedcentral.com/submit 